Research Article

\title{
Enhanced tethered-flight duration and locomotor activity by overexpression of the human gene SOD1 in Drosophila motorneurons
}

\author{
Agavni Petrosyan ${ }^{1}$, I-Hui Hsieh ${ }^{2}$, John P. Phillips ${ }^{3}$ and Kourosh Saberi ${ }^{1}$ \\ ${ }^{1}$ Department of Cognitive Sciences, University of California, Irvine, CA, USA. \\ ${ }^{2}$ Institute of Cognitive Neuroscience, National Central University, Jhongli City, Taiwan. \\ ${ }^{3}$ Department of Molecular Biology and Genetics, University of Guelph, Guelph, Ontario, Canada.
}

\begin{abstract}
Mutation of the human gene superoxide dismutase ( $h S O D 1)$ is associated with the fatal neurodegenerative disease familial amyotrophic lateral sclerosis (Lou Gehrig's disease). Selective overexpression of $h S O D 1$ in Drosophila motorneurons increases lifespan to $140 \%$ of normal. The current study was designed to determine resistance to lifespan decline and failure of sensorimotor functions by overexpressing $h S O D 1$ in Drosophila's motorneurons. First, we measured the ability to maintain continuous flight and wingbeat frequency (WBF) as a function of age (5 to 50 days). Flies overexpressing $h S O D 1$ under the D42-GAL4 activator were able to sustain flight significantly longer than controls, with the largest effect observed in the middle stages of life. The hSOD1-expressed line also had, on average, slower wingbeat frequencies in late, but not early life relative to age-matched controls. Second, we examined locomotor (exploratory walking) behavior in late life when flies had lost the ability to fly (age $\geq 60 \mathrm{~d}$ ). $h S O D 1$-expressed flies showed significantly more robust walking activity relative to controls. Findings show patterns of functional decline dissimilar to those reported for other life-extended lines, and suggest that the hSOD1 gene not only delays death but enhances sensorimotor abilities critical to survival even in late life.
\end{abstract}

Keywords: behavioral genetics, superoxide dismutase, Drosophila, motorneuron, longevity.

Received: April 30, 2014; Accepted: November 6, 2014.

\section{Introduction}

Drosophila melanogaster is an important animal model in the study of aging, life-extension, and functional decline, partly because of its short lifespan of approximately two months, which facilitates population-level studies of changes in behavior and physiology at various stages of life, and partly because of the high homology of its genes to other species including mammals (Rose et al., 1992; Curtsinger et al., 1995; Osiewacz, 1997; Parkes et al., 1998; Phillips et al., 2000). Comparative genomic studies, for example, have reported that of the hundreds of human disease genes examined, between 65 to $87 \%$ are conserved in Drosophila (Fortini et al., 2000; Reiter et al., 2001; Inlow and Restifo, 2004). Drosophila also displays a large repertoire of natural behaviors (visual, locomotor, olfactory, and auditory) that facilitate the study of sensorimotor decline as a function of aging.

The current study investigated age-dependent decline and failure of behavioral functions in transgenic Drosophila carrying the human gene superoxide dismutase ( $h S O D 1$ ). A gain-of-function mutation of the $h S O D 1$ gene

Send correspondence to Kourosh Saberi. Department of Cognitive Sciences, University of California, Irvine, CA, USA. E-mail: saberi@uci.edu. in humans is associated with the life-shortening paralytic disease Familial Amyotrophic Lateral Sclerosis (i.e., Lou Gehrig's disease; Rosen et al., 1993) which is characterized by gradual loss of motor functions, muscle fasciculation and atrophy, difficulty in speaking, breathing, and early death. Broad systemwide expression of human or endogenous SOD1 in Drosophila has no effect on longevity (Seto et al., 1990; Kirby et al., 2008). However, selective overexpression of hSOD1 in Drosophila's motorneurons increases longevity of healthy flies by $40 \%$ and rescues short-lived SOD1-null mutants to near-normal lifespan (Parkes et al., 1998). Increased longevity by hSOD1 overexpression is thought to occur by antioxidant intervention that mitigates cumulative DNA and cell damage caused by reactive oxygen species (Harman, 1956, 2003; Martin et al., 1996). This process is thought to be further mediated by hSOD1-triggered changes in signal transduction pathways, possibly through the neuroendocrine system, that regulate patterns of gene expression in a variety of aerobic cell types other than motorneurons (Phillips et al., 2000).

We describe here experiments on flight functions and locomotor activity of $h S O D 1$-expressed Drosophila. This is an important question for two reasons. First, an extended life devoid of healthy sensorimotor functions may not ex- 
perience a quality of life worth extending, and hence, determining the extent to which functional abilities are enhanced in life-extended lines is of value. Second, different lifeextending genes (e.g., hSOD1, Methuselah; INDY: I'm not dead yet) will likely yield different patterns of gerontological decline in sensory, motor, memory, and other behavioral functions. Findings from our earlier work on the age-dependent decline of functions in the Methuselah mutant differ markedly from those observed in the current study, which may be due to each gene's action on mechanisms subserving behavior, or to the patterns of a gene's expression in different tissues as we will describe later. These differing patterns of functional decline, nonetheless, suggest that multiple strategies to life extension may be useful in preserving distinct behavioral functions within an organism, strategies that may include different genetic as well as environmental approaches, e.g., drug consumption or calorie restriction (Sohal and Weindruch, 1996; Kang et al., 2002).

In the first part of the current study we examined the ability of $h S O D 1$-expressed and control flies to maintain continuous flight in a tethered-flight paradigm. We measured flight duration and wingbeat frequency (WBF) using an infrared laser coupled with a photodiode sensor system that recorded wingbeat time-series during phototaxic flight. We found that $h S O D 1$-expressed flies sustained flight longer than controls throughout much of their lifespan and had slower WBFs in late, but not early stages of life. In part two, we measured locomotor activity during late life in darkness and in a well-lit arena. $h S O D 1$ flies showed significantly more robust locomotor ability relative to controls. In addition, females outperformed males, but only for the $h S O D 1$ line, suggesting an interaction between genotype and sex in locomotor ability.

\section{Materials and Methods}

\section{Fly stocks}

hSOD1-expressed and control lines were generated at the University of Guelph as described fully in Parkes et al. (1998). Briefly, expression of a human SOD1 transgene in Drosophila motorneurons was achieved using the yeast GAL4/UAS system (Brand and Perrimon, 1993; Yeh et al., 1995; Gustafson and Boulianne, 1996). The D42-GAL4 activator used here is expressed broadly during embryogenesis, becomes restricted to motorneurons and interneurons during larval stages, and with the exception of a small number of unidentified neurons in the central brain, is restricted to motorneurons within the ventral ganglia in the adult fly. The hSOD 1 transgene consisted of a human SOD1 cDNA coupled to a yeast UAS element within a Drosophila P-transformation vector. Because both lifespan and behavior are affected by variation in genetic background, a number of genetic measures were taken in introducing the D42-GAL4 and UAS-hSOD1 transgenes into a uniform
Sod $+/+$ genetic background, and to construct expressing and non-expressing lines that were co-isogenic for most of the genome, with minimal differences in the genetic background between strains (see Parkes et al., 1998; Kirby et al., 2008). This allows tracing of behavioral phenotypes specifically to GAL4-activated $h S O D 1$ expression.

\section{Confirmation of life extension}

Virgin flies were sex-segregated within $4 \mathrm{~h}$ of eclosion and maintained in small laboratory vials (Genesee Scientific Corp.; polystyrene, O.D. x H 25 x 95 mm) containing fresh food media in a low-temperature incubator at $25{ }^{\circ} \mathrm{C}$ and $40 \%$ humidity on a 12/12 h dark/light cycle (VWR Scientific, Model 2015). They were transferred to fresh food vials every 3 to 4 days. We confirmed extension of the lifespan of $h S O D 1$-expressed flies relative to controls by approximately $30 \%$ in virgin females (68vs. 52 days) and 48\% in virgin males (65 vs. 44 days), measured at $50 \%$ mortality levels of a population of approximately 400 flies. Kaplan-Meier survival analyses (log rank Mantel-Cox test) showed that $h S O D 1$-expressed males significantly outlived control males $\left(\chi^{2}=119.1, \mathrm{p}<0.001\right)$ and $h S O D 1$-expressed females significantly outlived control females $\left(\chi^{2}=123.0, \mathrm{p}<0.001\right)$.

\section{Tethered-flight experiment}

Flight duration and wingbeat frequency were measured in a tethered-flight paradigm at $\sim 5 \mathrm{~h}$ after onset of subjective day. The tethering process involved several steps. First, an individual fly was lightly $\mathrm{CO}_{2}$ anesthetized and transferred to a custom-made aluminum block in a Peltier cooler (Boekel Scientific, Model 260014) on which a small opening $\left(2 \times 1 \times 1 \mathrm{~mm}^{3}\right)$ had been drilled to allow accurate positioning of an anesthetized fly (Figure 1). The fly remained under cold anesthesia at $4{ }^{\circ} \mathrm{C}$. Individual flies were gently handled either with a small brush or a jeweler's vacuum tweezers. The tip of a tungsten wire $(130 \mu$ in diameter) was dipped in glass glue (Loctite, New York, NY, USA), and under a stereomicroscope (Olympus SZ40) lowered using a micropositioner (Stoelting Co./Prior, England) onto the anesthetized fly's thorax. The glue was cured with a UV gun (Electro-Lite Corp., Model ELC-403) for $20 \mathrm{~s}$ and the fly was removed from the Peltier cooler using the micropositioner. Flies usually recovered from cold anesthesia and began flight within 3 to $4 \mathrm{~min}$. Tethered flies were moved to the experimental chamber, fed with a small piece of filter paper dipped in sucrose-water, and allowed to rest and become acclimated to the experimental environment for an additional 30-60 min prior to data collection. Prior work has shown that this is a sufficient period of time for full recovery from anesthesia (Lehmann and Dickinson, 1997; Petrosyan et al., 2007, 2013, 2014).

The tethered fly was positioned under a solid-state infrared (IR) laser (808 nm; Lasermate Group Inc., Pomona, Ca, USA, Model PLC8082AE) with an adjustable focus 

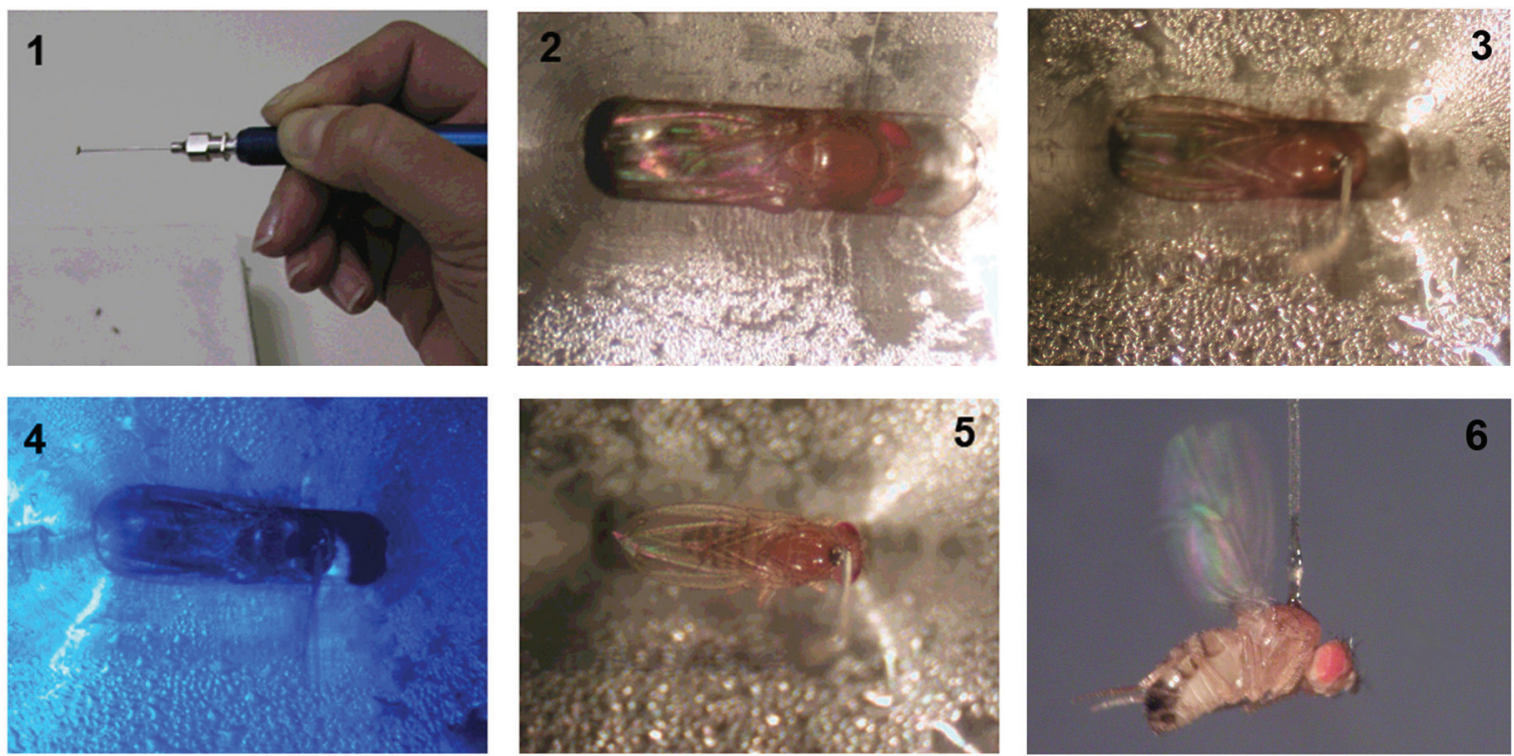

Figure 1 - Tethering a Drosophila for flight: 1) $\mathrm{A} \mathrm{CO}_{2}$ anesthetized fly was selected using vacuum tweezers, 2) positioned in a Peltier cooler at $4{ }^{\circ} \mathrm{C}$ which kept it under cold anesthesia, 3) a micropositioner was used to lower a tungsten wire dipped in glass glue onto its thorax, 4) cured with UV for approximately $20 \mathrm{~s}, 5$ ) removed from the Peltier cooler using the micropositioner, 6) The fly recovered from anesthesia in 3 to 4 min and began flight.

that cast shadows of the wing beats onto fast-response IR photodiode sensors (Photonic Detectors Inc., Simi Valley, CA, USA, Part no. PDB-C615-2). This setup is shown in Figure 2. The sensors were placed in a small plastic box covered with an IR bandpass filter (Edmund Industrial Optics, Barrington, NJ, USA, Part. no. NT32769). The experiment was run in complete darkness in a steel chamber $(2 \times 2$ x $2 \mathrm{~m}^{3}$; IAC) with only a single green LED $(555 \mathrm{~nm})$ posi-

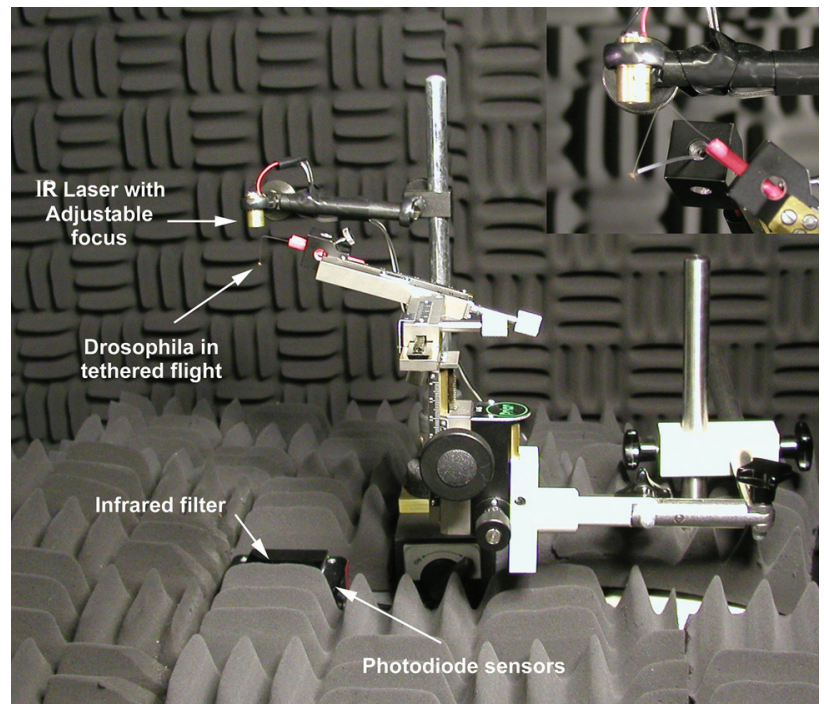

Figure 2 - Apparatus used for measurement of wingbeat frequency. A tethered fly was positioned under a solid-state infrared (IR) laser with an adjustable focus. Wingbeat shadows were cast onto IR photodiode sensors. The experiment was run in complete darkness in a steel chamber with only a single green LED $(555 \mathrm{~nm})$ positioned directly in the fly's line of sight to stimulate phototaxic flight. Inset shows the laser and acoustic microphone assembly (see text). tioned directly in the fly's line of sight at a distance of $15 \mathrm{~cm}$ to provide a visual target for phototaxis (Hadler, 1964; Miller et al., 1981).

The output of the photodiode sensors were sent to an amplifier and fed into an analog-to-digital converter (Sound Blaster Live, -120 dB noise floor) positioned outside the chamber, and recorded at a sampling rate of $10 \mathrm{kHz}$. WBF was measured during the first minute of flight after the rest period. Each fly's wing-beat data were saved in a digital file for later processing. An individual fly's WBF was determined as the average of five to ten one-second samples of flight. This sampling scheme has been shown to be sufficiently representative of the average WBF in phototaxic flight (Petrosyan et al., 2007). For each one-second sample, the wing-beat waveform was FastFourier transformed and the frequency corresponding to the peak of this function was determined as the WBF for that sample (i.e., 1-Hz frequency resolution).

To verify that WBF measured using the laser system is accurate, wing beats of one individual fly were recorded using the laser-system and, simultaneously, with a silicon probe-tube acoustic microphone (Figure 2 inset; Etymotic Research, Elk Grove Village, IL, USA ER-7C), fed to a digital-to-analog convertor (Sound Blaster Live) and digitized at a sampling rate of $10 \mathrm{kHz}$. The outputs of the two measurement systems were led to two separate computers. We found a near-perfect correlation between the acoustic measure of wing-beat waveform and laser measurements $(\mathrm{r}>0.99)$.

Flight performance was measured at four age categories of 5, 10,30, and 50 days for both genotypes, and additionally at the ages of 20 and $40 \mathrm{~d}$ for the $h S O D 1$ line. The 
age categories of 10 and 50 days represent groups of flies that were between 9 to 10 days of age, and 50 to 51 days of age respectively. After each experimental run, flies were $\mathrm{CO}_{2}$ anesthetized, removed from tether, and discarded in citrus oil.

\section{Locomotor (walking) behavior}

The distance walked in a fixed period of time has previously been used as a measure of exploratory behavior and locomotor ability in Drosophila (Strauss et al., 1992; Hayward et al., 1993; Roberts, 1998). The current experiment measured average walking distance for both genotypes in a large arena $(60 \times 45 \mathrm{~cm})$. Since the main goal of the experiments was to examine sensorimotor senescence at old age, the minimum age tested was 60 days. At this age, flies cannot sustain their weight in free flight and therefore nearly always walk to explore their environment. On the day of the experiment, none of the flies were anesthetized. A total of 161 flies were tested (100 females and 61 males), with an average of approximately 12 flies per age group for each genotype. Flies were gently positioned in a $2.54 \mathrm{~cm}^{2}$ (1-inch) square grid drawn in the center of the arena and the distance that they moved away from the center square in a 5 -min period was used as a measure of locomotor activity. The experiment was run either in a well-lit arena or in complete darkness, recorded by an infrared camera, and walking distance was determined after the session from the video recordings. If a fly did not move out of the center square, the distance traveled was recorded as zero. Male and female flies were run in separate sessions.

\section{Results}

\section{Tethered-flight experiment}

Figure 3 shows duration of the longest segment of sustained flight measured during the first minute of flight after rest for $h S O D 1$ and its parental strain. The $h S O D 1$ data are based on 28 individually tethered flies and the data from the parental control group are based on 25 flies. Flight duration generally declines as a function of age for both genotypes. Female $h S O D 1$ flies maintain flight significantly longer than female controls throughout most of their lifespan, but not at the oldest age measured (50 days). The convergence of sustained flight durations at late life may possibly represent a floor effect, where all flies were near the end of their ability to maintain flight. No significant differences were observed in the duration of sustained flight between male $h S O D 1$ and male control flies. Female $h S O D 1$ flies also sustained flight longer than male $h S O D 1$ flies, but no differences in flight duration were observed as a function of age between male and female control flies

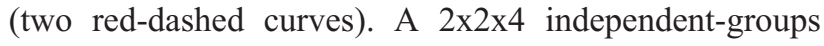
ANOVA showed a significant effect of genotype $\left(\mathrm{F}_{(1,37)}=\right.$ $6.49, \mathrm{p}<0.05)$, a significant effect of $\operatorname{sex}\left(\mathrm{F}_{(1,37)}=4.43\right.$, $\mathrm{p}<0.05)$, a significant effect of age $\left(\mathrm{F}_{(3,37)}=7.67\right.$,
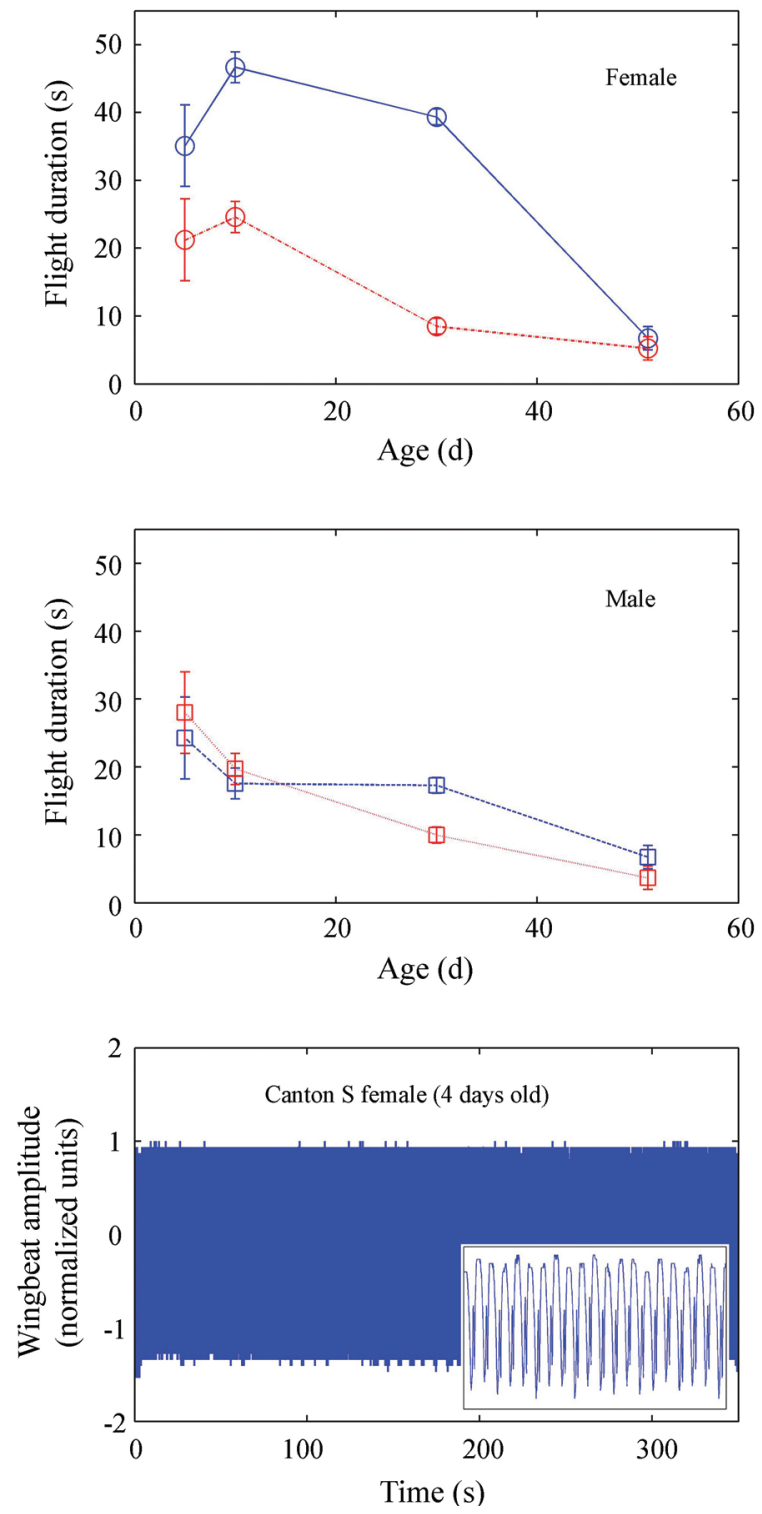

Figure 3 - Duration of the longest segment of sustained flight as a function of age and genotype. Top panel shows data for females and middle panel for males. Error bars represent one standard error. Bottom panel shows an extended uninterrupted flight of $5 \mathrm{~min}$ (approximately 50,000 wingbeats) by a 4-day old female Canton-S wild-type fly measured using the same apparatus and procedures employed in the current study. hSODl and their parental controls maintain flight at considerably shorter durations than wild types. Inset shows a 120 millisecond segment (20 wingbeats) of the waveform in the bottom panel.

$\mathrm{p}<0.001)$, and significant interaction between genotype and $\operatorname{sex}\left(\mathrm{F}_{(1,37)}=4.98, \mathrm{p}<0.05\right)$. No other interaction terms were significant.

Figure 4 shows averaged WBFs as a function of age for the same flies as those shown in Figure 3. WBF for both genotypes declined monotonically as a function of age, but was lower for $h S O D 1$ flies at older ages. A $2 \times 2 \times 4$ independent-groups ANOVA showed a significant effect of genotype: $F_{(1,42)}=6.34, p<0.05$, a significant effect of age: 

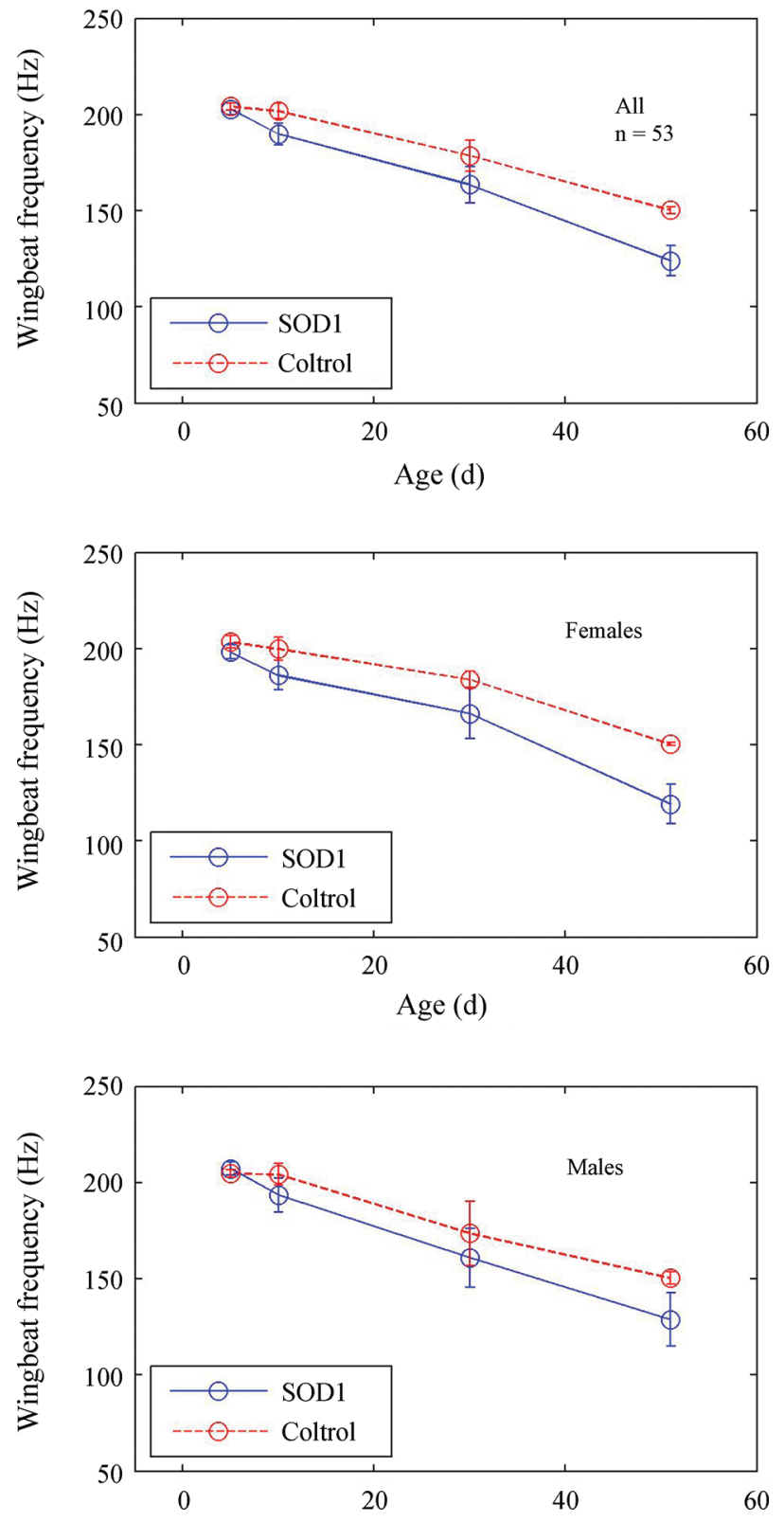

Figure 4 - Wingbeat frequency as a function of age for the $h S O D 1$ and control groups (top panel). Middle and bottom panels show data for female and male flies respectively. Error bars represent one standard error.

$\mathrm{F}_{(3,42)}=26.68, \mathrm{p}<0.001$, but no significant effect of sex: $\mathrm{F}_{(1,42)}=0.135$, n.s., and no significant interaction effects. The effect of genotype on WBF appears to result largely from differences in later stages of life. Post-hoc t-test analysis showed no significant genotype differences in WBFs at the earliest ages tested $\left(t_{(10)}=0.395\right.$, n.s. and $t_{(17)}=1.46$, n.s. for 5 and 10 day olds respectively), whereas a statistically significant genotype difference was observed for the oldest group $\left(\mathrm{t}_{(9)}=2.90, \mathrm{p}<0.05\right)$.

\section{Locomotion (walking) experiment}

Figure 5 shows results of the late-life locomotor behavior experiment. The average distance that $h S O D 1$ and control groups had moved away from the center square in a 5 -min period is shown as a function of age. This distance was measured from video recordings in both the well-lit and dark (via infrared) conditions. The left panels are data for males and the right panels for females. Black and white bars, respectively represent data collected in complete darkness and in the well-lit condition. A number of trends are evident. First, as expected, the level of locomotor activity declines with age. Second, there is generally a greater level of activity in the well-lit condition, though this advantage is less evident for the very oldest age groups. The hSOD1 flies are clearly more active compared to controls (compare activity at age $63 \mathrm{~d}$ ). The $h S O D l$ females were the most active of all groups at all age categories. Data were not collected for the control group at ages 67 and higher because none had survived till that age.

We conducted a $2 \times 2 \times 2$ mixed-design ANOVA on the locomotor data of one age group (63 d) at which we had data across genotype, sex, and lighting condition. Genotype and sex served as the between-subjects variables and "lighting condition" as the repeated measures variable because locomotor activity of the same flies were measured in both dark and light conditions. We found a significant effect of lighting condition: $\mathrm{F}_{(1,52)}=8.43, \mathrm{p}<0.01$, a significant effect of genotype: $F_{(1,52)}=15.79, p<0.001$, but no significant effect of sex: $F_{(1,52)}=3.64$ (though this effect did approach significance at $\mathrm{p}=0.062$ ). Several interaction terms were also significant, including the interaction between genotype and sex: $\left.F_{(1,52)}=7.07, p<0.05\right)$, between lighting condition and sex: $\mathrm{F}_{(1,52)}=5.94, \mathrm{p}<0.05$, and the 3 -way interaction between genotype, sex, and lighting: $\mathrm{F}_{(1,52)}=5.52, \mathrm{p}<0.05$.

\section{Discussion}

The experiments described here provide data on functional abilities of a transgenic line of Drosophila melanogaster whose lifespan has been extended by overexpression of the human gene $h S O D 1$ in motorneurons. We found that female $h S O D 1$ flies were able to sustain continuous flight longer than control females. The effect was largest during middle stages of life between 10 and 30 days of age (Figure 3). No significant difference in the ability to maintain continuous flight was observed between the male hSOD 1 and male control flies. Both genotypes ( $h S O D 1$ and controls) generally sustained flight for shorter durations compared to wild types previously tested using similar (e.g., tethered; Lehmann and Dickinson, 1997) or different experimental paradigms (e.g., free-flight box experiments; Miller et al., 2008). For comparison, we measured the duration of sustained tethered flight for a 4-day old female Canton-S wild-type fly using the exact same apparatus and preparation protocol employed in our study of $h S O D 1$ flies. Bottom panel of Figure 3 shows that this genotype maintains flight significantly longer than $h S O D l$ and parental controls. The figure shows an extended uninterrupted flight 

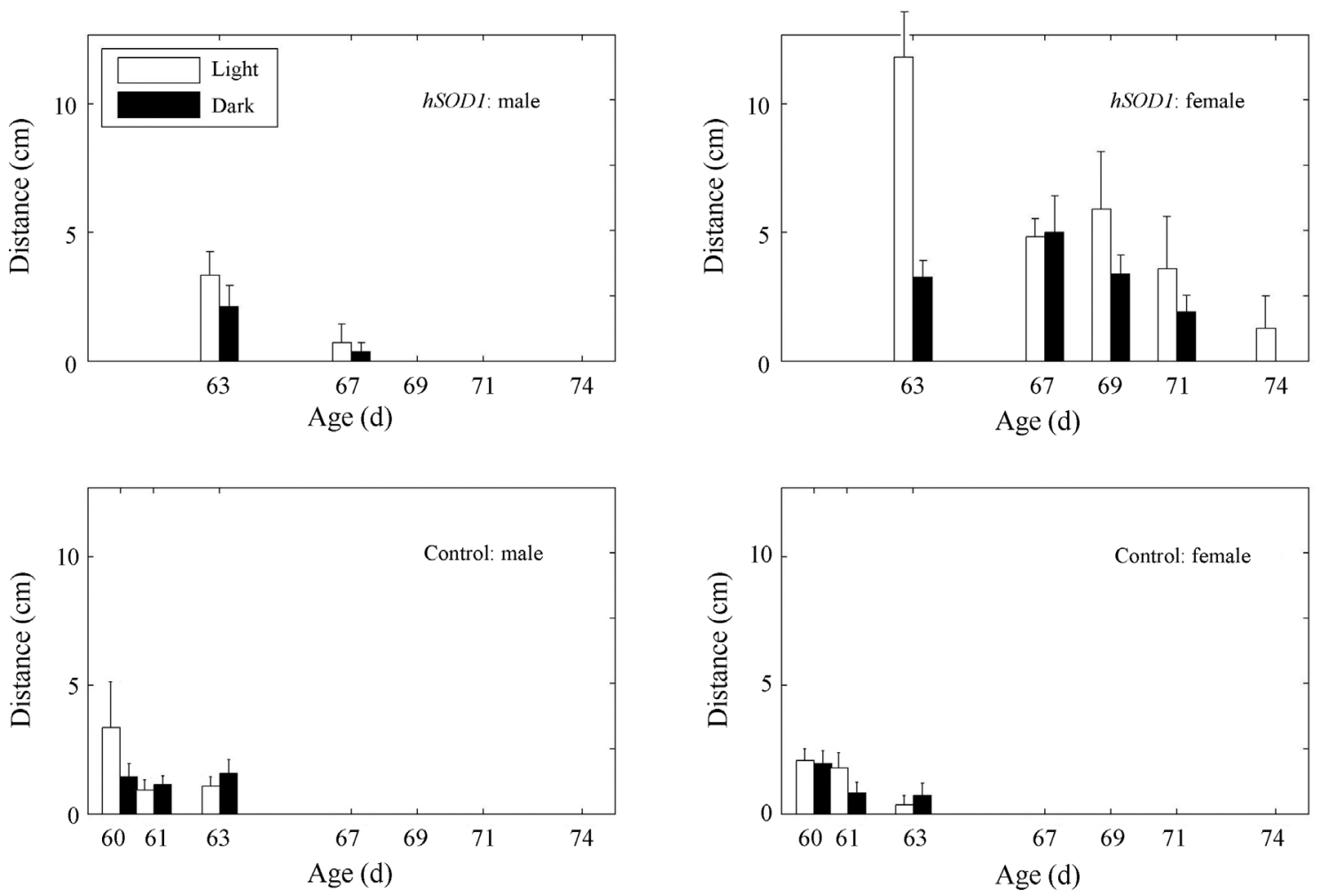

Figure 5 - Distance walked as a measure of locomotor activity for the $h S O D 1$ (top panels) and control groups (bottom) as a function of age. Left panels show data from males and right panels for females. Black bars represent activity measured in complete darkness, and white bars under well-lit conditions. Error bars represent one standard error.

of 5 min (approximately 50,000 wingbeats). Inset shows a 120 millisecond segment (20 wingbeats) of the 5-min waveform. The reason for this difference in flight duration ability between wild types and $h S O D 1$ flies is not clear and may be due to differences in motor output efficiency, levels of metabolic activity, or other genetic and physiological factors.

An additional finding was a small but statistically significant lower wingbeat frequency for $h S O D 1$ flies in middle and late, but not early life relative to controls. It is unlikely, however, that this lower wingbeat frequency signifies a reduced level of metabolic activity since levels of locomotor (walking) activity at old age were significantly higher for $h S O D 1$ than control flies. In addition, measures of respiration rate in a prior study suggest equivalent levels of oxygen consumption for $h S O D 1$ and control flies, even in late life (Parkes et al., 1998). A lower wingbeat frequency does not necessarily signal a disadvantage in flight ability, but may potentially signify improved motor output efficiency, especially in light of the observed longer sustained flight in these same flies. Flight velocity for some species (e.g., bats and birds) has been shown to be negatively correlated with wingbeat frequency for a wide range of velocities (Bullen and McKenzie, 2002; Tobalske et al., 2003), supporting the idea that a slower wingbeat frequency may allow use of less energy in sustaining highervelocity and longer distance/duration flights. While we have not measured wing-stroke amplitude to determine if total aerodynamic power (a nonlinear product of wingbeat frequency and stroke amplitude) has increased or decreased as a result of overexpressing $h S O D 1$, the increase in flight duration itself suggests that at least this aspect of sustained-flight has been enhanced, likely providing an adaptive advantage for survival. Furthermore, we have also noticed that the wingbeat frequencies of some Drosophila genotypes that maintain flight duration considerably longer than the $h S O D l$ (and control) flies are lower than the latter genotypes. For example, the average wingbeat frequency of the Canton-S fly shown in the bottom panel of Figure 3 measured in identical conditions (temperature, humidity, lighting, diet, etc.) to those used in the current study is approximately $160 \mathrm{~Hz}$ as compared to over $200 \mathrm{~Hz}$ for $h S O D 1$ and controls at a similar age category (4 vs. 5 day olds).

The observed improvements in flight duration and locomotor ability are likely due to enhanced antioxidant defense and resistance of cells, especially motorneurons, to cumulative damage caused by reactive oxygen species. These improvements may also partly be related to secondary mechanisms, for example, through $h S O D 1$-triggered changes in signal transduction pathways that regulate gene expression in other cell types. Our results, however, also suggest that flight and locomotor systems are affected differently by $h S O D 1$ overexpression, at least in late life. We observed no differences in flight duration and a signifi- 
cantly lower wingbeat frequency for $h S O D 1$ flies relative to controls at late life. Conversely, we observed significantly higher levels of locomotor activity in late life by hSOD1 overexpression.

Female flies in our study showed a significant effect of $h S O D 1$ overexpression on flight duration, while no effect was observed for males. Locomotor abilities, however, were improved for both, though still significantly more for females. Why there may be a larger effect of $h S O D 1$ overexpression on functional abilities of female Drosophila compared to males is not clear. Female Drosophila of a number of genotypes have previously been shown to have more robust functional abilities, better survival rates, and more resistance to toxicity than males, likely, at least in part, due to their significantly larger bodies. Perhaps the more robust abilities of female Drosophila translates into more effective motor function improvements in response to hSOD1 overexpression. There is, in addition, evidence from the mouse ALS model that female SOD1 mice respond more effectively to drug treatment (methionine sulfoximine; MSO) than do males in mitigating the effects of motorneuron degeneration as measured by a significant improvement in maintaining muscle grip on a wire grid (Bame et al., 2012).

A comparison of age-dependent changes in behavioral functions reported here to that reported for a different life-extended mutant, the Methuselah (Lin et al., 1998), reveals markedly different patterns of functional decline. Petrosyan et al. (2007) have shown that the $m t h$ mutant has a higher wingbeat frequency relative to its parental control group throughout most of its lifespan, but especially during early and middle stages of life. This finding is opposite to that observed for the $h S O D 1$ line which displays a lower wingbeat frequency than its parental line throughout most of its lifespan, and equal WBF during early life. Locomotor abilities of the $m$ th flies also decline in a different pattern compared to that of $h S O D 1$-expressed flies. $m t h$ flies show a lower level of locomotor activity relative to controls in late life. $h S O D 1$-expressed flies, however, show higher levels of locomotion relative to controls at old age. Interestingly, the INDY life-extended mutant has been reported to display even a third pattern, with no difference observed in locomotor activity (negative geotaxis) relative to control flies in late life (Martin and Grotewiel, 2006).

One should, however, be cautious in interpreting patterns of behavioral change across genotypes. Such differences may be due to the consequence of each gene's action on functional mechanisms subserving a specific behavior, or they may be due to selectivity of expression within different tissues, or due to other complex factors. In $h S O D 1$ flies, for example, expression is restricted primarily to motorneurons and a small number of other cell types, whereas the $m t h$ gene is expressed broadly, and INDY is primarily expressed on cell membranes of the midgut and the plasma membranes of fat body and oenocytes (Knauf et al.,
2002). Furthermore, the $h S O D 1$ gene is not expressed in all motorneurons and may itself mediate changes in signal transduction pathways that regulate patterns of gene expression in cell types other than motorneurons (Phillips et al., 2000). Nonetheless, such differences in enhancement of behavioral abilities do exist across life-extended genotypes, and are both informative and significant.

We should also caution that the enhanced functions observed are a result of overexpressing the normal human SOD1 gene, whereas familial ALS is associated with a mutation of SOD1. There is evidence that the most common type of ALS-related SOD1 mutation results in a gain (not loss) of function that leads to induced motorneuron toxicity and eventual death (Bruijn et al., 1998). Overexpressing the normal $h S O D 1$, however, is likely to enhance antioxidant resistance to free radicals (and toxicity) whose accumulative effects may cause motorneuron dysfunction and death (Parkes et al., 1998). We should also caution that extension of the current findings to therapeutic strategies for human ALS is complex and challenging, as prior attempts to enhance antioxidant defenses in humans afflicted with ALS have had mixed results due to a number of factors such as dosage, safety, and different pharmacokinetic effects across species (Lanka and Cudkowicz, 2008).

In summary, we have found that for a simple organism, expression of the human gene $h S O D 1$ in motorneurons enhances sensorimotor functions at different ages. These include simple motor functions such as flight duration at early and middle life, and locomotor activity in late life. A comparison of the current results to our earlier studies (Petrosyan et al., 2007, 2013, 2014) suggests that different life-extending genes may yield different patterns of functional stability at various stages of life. Whether these different genetic strategies may be used in concert to broadly promote robust motor, sensory, memory, and other functions in late life, merits further investigation.

\section{Acknowledgments}

We thank Rosana Magalhães, Eugénia Fernandes, and Jorge Alves for helpful discussions, and Michael Dickinson for technical suggestions. We also thank three reviewers for their helpful comments. Work supported by funding from the University of California.

\section{References}

Bame M, Pentiak PA, Needleman R and Brusilow WSA (2012) Effect of sex on lifespan, disease progression, and the response to methionine sulfoximine in the SOD1 G93A mouse model for ALS. Gender Med 9:524-535.

Bruijn LI, Houseweart MK, Kato S, Anderson KL, Anderson SD, Ohama E, Reaume AG, Scott RW and Cleveland DW (1998) Aggregation and motor neuron toxicity of an ALSlinked SOD1 mutant independent from wild-type SOD1. Science 281:1851-1854. 
Brand AH and Perrimon N (1993) Targeted gene expression as a means of altering cell fates and generating dominant phenotypes. Development 118:401-415.

Bullen RD and McKenzie NL (2002) Scaling bat wingbeat frequency and amplitude. J Exp Biol 205:2615-2626.

Curtsinger JW, Fukui HH, Khazaeli AA, Kirscher A, Pletcher SD, Promislow SEL and Tatar M (1995) Genetic variation and aging. Annu Rev Genet 29:553-575.

Gustafson K and Boulianne GL (1996) Distinct expression patterns detected within individual tissues by the GAL4 enhancer trap technique. Genome 39:174-182.

Fortini ME, Skupski MP, Boguski MS and Hariharan IK (2000) A survey of human disease gene counterparts in the Drosophila genome. J Cell Biol 150:F23-F29.

Hadler NM (1964) Genetic influence on phototaxis in Drosophila melanogaster. Biol Bull 126:264-273.

Harman D (1956) Aging - A theory based on free-radical and radiation-chemistry. J Gerontol 11:298-300.

Harman D (2003) The free radical theory of aging. Antioxid Redox Signal 5:557-561.

Hayward DC, Delaney SJ, Campbell HD, Ghysen A, Benzer S, Kasprzak AB, Cotsell JN, Young IG and Miklos GLG (1993) The sluggish-A gene of Drosophila melanogaster is expressed in the nervous system and encodes proline oxidase, a mitochondrial enzyme involved in glutamate biosynthesis. Proc Natl Acad Sci USA 90:2979-2983.

Inlow JK and Restifo LL (2004) Molecular and comparative genetics of mental retardation. Genetics 166:835-881.

Kang HL, Benzer S and Min KT (2002) Life extension in Drosophila by feeding a drug. Proc Natl Acad Sci USA 99:838-843.

Knauf F, Rogina B, Jiang Z, Aronson PS and Helfand SL (2002) Functional characterization and immunolocalization of the transporter encoded by the life-extending gene Indy. Proc Natl Acad Sci USA 99:14315-14319.

Kirby K, Jensen LT, Binnington J, Hilliker AJ, Ulloa J, Culotta VC and Phillips JP (2008) Instability of superoxide dismutase 1 of Drosophila in mutants deficient for its cognate copper chaperone. J Biol Chem 283:35393-35401.

Lanka V and Cudkowicz M (2008) Therapy development for ALS: Lessons learned and path forward. Amyotroph Lateral Scler 9:131-140.

Lehmann FO and Dickinson MH (1997) The changes in power requirements and muscle efficiency during elevated force production in the fruit fly Drosophila melanogaster. J Exp Biol 200:1133-1143.

Lin YJ, Seroude L and Benzer S (1998) Extended life-span and stress resistance in the Drosophila mutant methuselah. Science 282:943-946.

Martin GM, Austad SN and Johnson TE (1996) Genetic analysis of ageing: Role of oxidative damage and environmental stresses. Nat Genet 13:25-34.

Martin I and Grotewiel MS (2006) Distinct genetic influences on locomotor senescence in Drosophila revealed by a series of metrical analyses. Exp Geront 41:877-881.

Miller GV, Hansen KN and Stark WS (1981) Phototaxis in Drosophila - R1-6 input and interaction among ocellar and compound eye receptors. J Insect Physiol 27:813-819.
Miller MS, Lekkas P, Braddock JM, Farman GP, Ballif BA, Irving TC, Maughan DW and Vigoreaux JO (2008) Aging enhances indirect flight muscle fiber performance yet decreases flight ability in Drosophila. Biophys J 95:23912401.

Osiewacz HD (1997) Genetic regulation of aging. J Mol Med 75:715-727.

Parkes TL, Elia AJ, Dickinson D, Hilliker AJ, Phillips JP and Boulianne GL (1998) Extension of Drosophila lifespan by overexpression of human SOD1 in motorneurons. Nat Genet 19:171-174.

Petrosyan A, Hsieh I and Saberi K (2007) Age-dependent stability of sensorimotor functions in the life-extended Drosophila mutant methuselah. Behav Genet 37:585-594.

Petrosyan A, Hsieh I, Phillips JP and Saberi K (2013) Enhanced optomotor efficiency by expression of the human gene superoxide dismutase primarily in Drosophila motorneurons. J Neurogenet 27:59-67.

Petrosyan A, Goncalves OF, Hsieh I and Saberi K (2014) Improved functional abilities of the life-extended Drosophila mutant Methuselah are reversed at old age to below control levels. Age 36:213-221.

Phillips JP, Parkes TL and Hilliker AJ (2000) Targeted neuronal gene expression and longevity in Drosophila. Exp Gerontol 35:1157-1164.

Reiter LT, Potocki L, Chien S, Gribskov M and Bier E (2001) A systematic analysis of human disease-associated gene sequences in Drosophila melanogaster. Genome Res 11:1114-1125.

Roberts DB (1998) Drosophila: A Practical Approach. $2^{\text {nd }}$ edition. University Press, New York, 416 pp.

Rose MR, Vu LN, Park SU and Graves JL (1992) Selection on stress resistance increases longevity in Drosophila melanogaster. Exp Gerontol 27:241-250.

Rosen DR, Siddique T, Patterson D, Figlewicz DA, Sapp P, Hentati A, Donaldson D, Goto J, O'Reagan JP, Deng HX, et al. (1993) Mutations in $\mathrm{Cu} / \mathrm{Zn}$ superoxide dismutase gene are associated with familial amyotrophic lateral sclerosis. Nature 362:59-62.

Seto NO, Hayashi S and Tener GM (1990) Overexpression of $\mathrm{Cu}-\mathrm{Zn}$ superoxide dismutase in Drosophila does not affect life-span. Proc Natl Acad Sci USA 87:4270-4274.

Sohal RS and Weindruch R (1996) Oxidative stress, caloric restriction, and aging. Science 273:59-63.

Strauss R, Hanesch U, Kinkelin M, Wolf R and Heisenberg M (1992) No-bridge of Drosophila melanogaster - Portrait of a structural brain mutant of the central complex. J Neurogenet 8:125-155.

Tobalske BW, Hedrick TL, Dial KP and Biewener AA (2003) Comparative power curves in bird flight. Nature 421:363365 .

Yeh E, Gustafson K and Boulianne GL (1995) Green fluorescent protein as a vital marker and reporter of gene expression in Drosophila. Proc Natl Acad Sci USA 92:7036-7040.

\section{Associate Editor: Carlos F.M. Menck}

License information: This is an open-access article distributed under the terms of the Creative Commons Attribution License, which permits unrestricted use, distribution, and reproduction in any medium, provided the original work is properly cited. 